\title{
Valsartan: come versatilità \\ e tollerabilità possono contribuire \\ al contenimento della spesa sanitaria
}

Francesco Vittorio Costa ${ }^{(1)}$

\begin{abstract}
The search for a more specific and complete blockade of the hypertensive effects of angiotensin and of better tolerability than ACE-inhibitors has led to the development of angiotensin II receptor blockers (ARBs). ARBs have been evaluated in several large studies in terms of efficacy and safety in reducing blood pressure, as well as for cardiovascular and renal protection. Among angiotensin II receptor blockers, valsartan and losartan have the greatest number of therapeutic indications. In light of the costs related to cardiovascular disorder valsartan represents a potential long-term health cost saving strategy. Furthermore valsartan shows one of the lowest costs for patient achieving blood pressure goals and the higher level of persistence in its class.
\end{abstract}

Keywords: valsartan, angiotensin II receptor blockers, health cost

Farmeconomia e percorsi terapeutici 2011; 12(Suppl 1): 9-17

\section{INTRODUZIONE}

Sono ormai trascorsi più di 15 anni dalla commercializzazione di losartan, il primo bloccante dei recettori dell'angiotensina II (comunemente chiamati sartani) e da allora altre sei mo- lecole si sono rese disponibili in Italia. Questa famiglia di farmaci ha migliorato le possibilità terapeutiche in patologie molto diffuse e gravi quali l'ipertensione arteriosa, lo scompenso cardiaco, l'infarto del miocardio, la nefropatia (l) Casa Di Cura Madre Fortunata Toniolo, Bologna

Corresponding author Francesco Vittorio Costa fvcosta@libero.it

\begin{tabular}{|c|c|c|c|c|c|c|c|}
\hline Indicazione & Lo & Epro & Val & Irbe & Cande & Telmi & Olme \\
\hline Ipertensione & $\mathrm{x}$ & $\mathrm{x}$ & $x^{\star}$ & $\mathrm{x}$ & $\mathrm{x}$ & $\mathrm{x}$ & $x$ \\
\hline $\begin{array}{l}\text { Pz ipertesi con IVS per ridurre il rischio di mortalità e morbilità cv rappresentato } \\
\text { prevalentemente dal rischio di ictus }\end{array}$ & $x$ & & & & & & \\
\hline $\begin{array}{l}\text { Trattamento dell'insuff. cardiaca, quando il trattamento con un ACE inibitore } \\
\text { non è tollerato o è controindicato }\end{array}$ & $x$ & & $x$ & & $x$ & & \\
\hline $\begin{array}{l}\text { Trattamento dell'insuff. cardiaca e alterata funzione sistolica sx in aggiunta al } \\
\text { trattamento con un ACE inibitore }\end{array}$ & & & & & $x$ & & \\
\hline $\begin{array}{l}\text { Trattamento dell'insuff. cardiaca sintomatica come terapia aggiuntiva agli ACE } \\
\text { inibitori quando non possono essere usati i } \beta \text {-bloccanti }\end{array}$ & & & $x$ & & & & \\
\hline $\begin{array}{l}\text { Infarto del miocardio: trattamento di pz clinicamente stabili con insuff. cardiaca } \\
\text { sintomatica o disfunzione sistolica ventricolare sx asintomatica secondaria a } \\
\text { infarto miocardico recente (12h-10gg) }\end{array}$ & & & $x$ & & & & \\
\hline $\begin{array}{l}\text { Trattamento della malattia renale nei pz ipertesi con diabete mellito di tipo } 2 \\
\text { come parte di un trattamento antipertensivo }\end{array}$ & & & & $x$ & & & \\
\hline $\begin{array}{l}\text { Trattamento della patologia renale in pz con ipertensione e diabete mellito di } \\
\text { tipo } 2 \text { con proteinuria }>0,5 \mathrm{~g} / \text { die nel contesto di una terapia antipertensiva }\end{array}$ & $x$ & & & & & & \\
\hline Riduzione del rischio di ictus in pz ipertesi con IVS documentata con ECG & $x$ & & & & & & \\
\hline Trattamento dell'ipertensione essenziale in bambini e adolescenti di 6-12 anni & $x$ & & & & & & \\
\hline $\begin{array}{l}\text { Prevenzione cv. Riduzione della mortalità cv in pz con: } \\
\text { - Malattia cv aterotrombotica manifesta (storia di coronaropatia, ictus o } \\
\text { malattia arteriosa periferica) } \\
\text { - Diabete mellito di tipo } 2 \text { con danno documentato negli organi bersaglio }\end{array}$ & & & & & & $x$ & \\
\hline
\end{tabular}

\section{Tabella I}

Indicazioni dei sartani in commercio in Italia. Tutte le indicazioni sono rimborsate dal SSN a eccezione di telmisartan nella prevenzione cv; tale indicazione, sebbene presente in scheda tecnica, non è infatti attualmente rimborsata dal SSN

* esclusa dose $40 \mathrm{mg}$

Cande = candesartan; Epro = eprosartan; Irbe = irbesartan; IVS = ipertrofia ventricolare sinistra; Lo = losartan; OIme = olmesartan; Telmi $=$ telmisartan; $\mathrm{Val}=$ valsartan 
diabetica [1-4] e ha avuto un immediato successo soprattutto perché i sartani, oltre ad avere un'efficacia almeno pari a quella degli altri antipertensivi, hanno un'eccezionale tollerabilità, sovrapponibile a quella del placebo.

Come vedremo in seguito, questa caratteristica è molto importante in quanto garantisce tassi di aderenza e persistenza al trattamento molto migliori rispetto alle altre famiglie di farmaci antipertensivi.

Nel corso degli ultimi anni sono stati portati a termine diversi studi controllati con i sartani e, sulla base dei risultati di alcuni di questi, sono state poste le indicazioni al loro utilizzo in molte situazioni cliniche. A parte l'ipertensione arteriosa, che è comune a tutti i sartani, le indicazioni differiscono da molecola a molecola (Tabella I). Il capostipite della famiglia, losartan, è insieme a valsartan quello che ha più indicazioni cliniche ma un'analisi attenta della letteratura mostra che esistono differenze importanti tra i due farmaci.

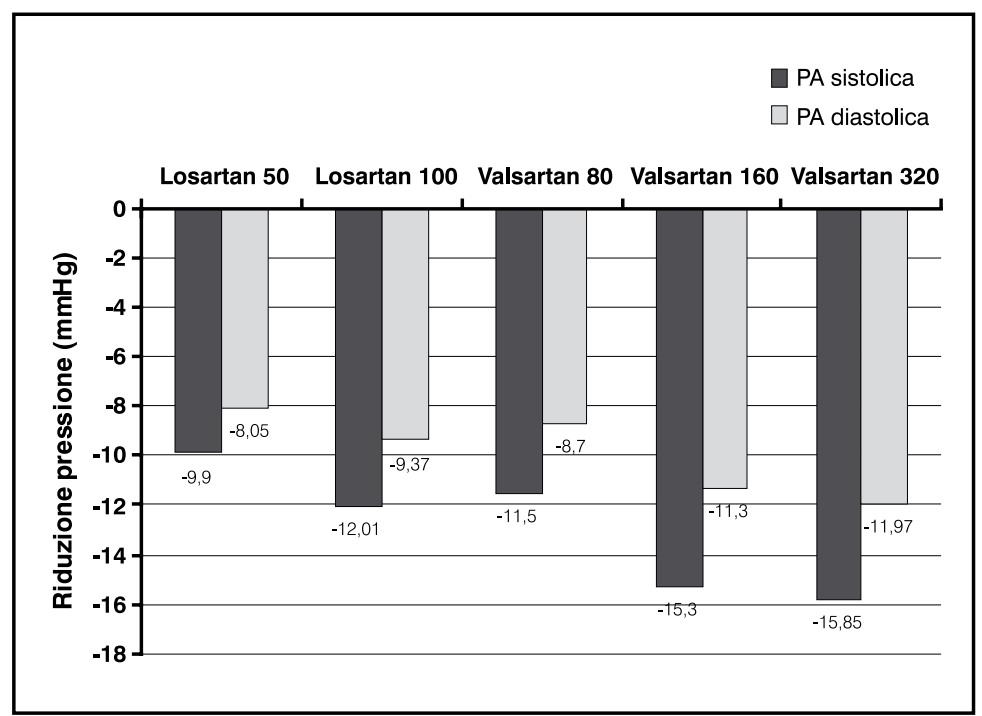

Figura 1

Riduzione della PA sistolica e della PA diastolica ottenuta con varie dosi di losartan e valsartan (modificato da Lawes, 2004 [6])

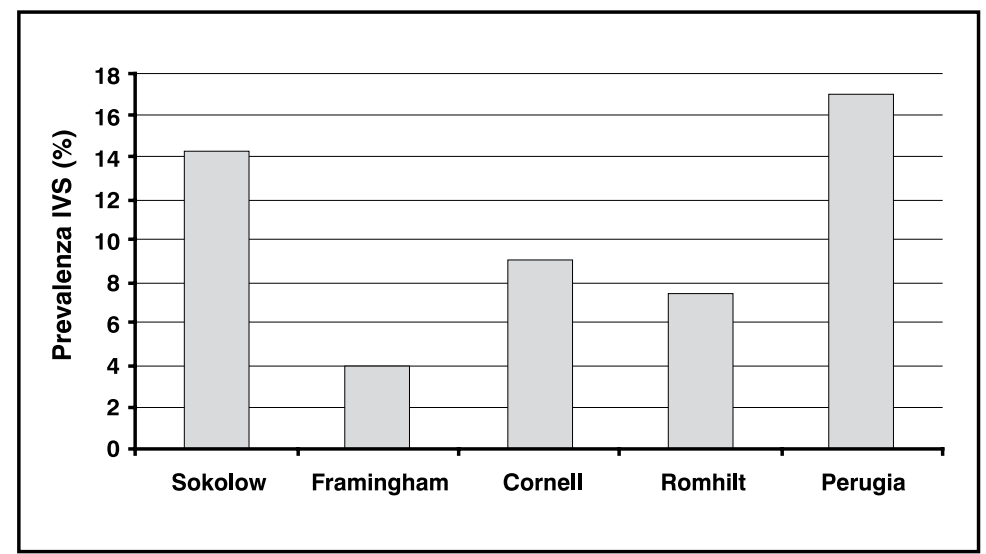

Figura 2

Prevalenza di IVS rilevata a seconda dei differenti criteri elettrocardiografici utilizzati (modificata da Schillaci, 2000 [8])

\section{EFFICACIA CLINICA: VALSARTAN VS LOSARTAN}

\section{Ipertensione arteriosa}

Una metanalisi del 2009 [5] ha mostrato che l'efficacia antipertensiva di losartan $50 \mathrm{mg} / \mathrm{die}$ è inferiore a quella di valsartan somministrato a 80,160 o $320 \mathrm{mg} /$ die (Figura 1). Alla dose di $100 \mathrm{mg} /$ die, losartan è efficace quanto la dose più bassa di valsartan $(80 \mathrm{mg} / \mathrm{die})$, ma è meno efficace di valsartan 160 e $320 \mathrm{mg} /$ die; la differenza di calo pressorio raggiunge poco meno di $4 \mathrm{mmHg}$ per la sistolica e $2,5 \mathrm{mmHg}$ per la diastolica. Tali differenze sono assai rilevanti dal punto di vista clinico dato che una riduzione di $5 \mathrm{mmHg}$ della PA sistolica, comporta una diminuzione del rischio di ictus del 15\% [6].

\section{Ipertrofia ventricolare sinistra}

Lo studio LIFE [7] ha fornito le basi per l'indicazione di losartan in pazienti ipertesi con ipertrofia ventricolare sinistra (IVS) che costituiscono un gruppo di soggetti che, avendo un danno d'organo, sono a elevato rischio cardiovascolare. Losartan è stato confrontato con atenololo ed è risultato superiore nel prevenire l'endpoint primario $(23,8 \%$ vs $27,9 \%$; $\mathrm{p}<0,02)$ e nel ridurre l'incidenza di ictus (-25\%).

Tuttavia occorre rilevare che nello studio LIFE l'IVS veniva diagnosticata mediante ECG la cui utilità pratica come strumento di stratificazione prognostica è limitata dalla bassa prevalenza di IVS all'ECG nei soggetti con ipertensione arteriosa (Figura 2). L'ipertrofia classica (con presenza simultanea di alti voltaggi e di alterazioni della ripolarizzazione) è infatti presente in una percentuale oscillante tra il $5 \%$ ed il $15 \%$ nei soggetti ipertesi [8]; l'informazione che l'ECG veicola è quindi limitata a un'esigua minoranza di soggetti e i pazienti arruolati nello studio LIFE sono poco rappresentativi della popolazione generale di ipertesi.

Valsartan è stato valutato in pazienti ad alto rischio cardiovascolare in tre studi: VALUE, JIKEI e KYOTO. Lo studio VALUE [9] ha analizzato oltre 15.000 pazienti ipertesi (trattati e non) ad alto rischio cardiovascolare e ha mostrato che valsartan è efficace nel prevenire gli eventi come amlodipina. Tuttavia i soggetti randomizzati ad amlodipina, avevano valori pressori più bassi e ciò fa sicuramente sottostimare l'efficacia sugli eventi di valsartan e ipotizzare che esso abbia effetti protettivi a prescindere dall'impatto sulla pressione arteriosa. Le reazioni avverse sono risultate meno frequenti con valsartan e i pazienti trattati con esso hanno sviluppato diabete in misura significativamente minore e hanno subìto meno ricoveri per scompenso cardiaco, rispetto ad amlodipina. Lo studio JIKEI [10] ha studiato 3.081 pazienti giapponesi già in trattamento per ipertensione, coronaropatie o scompenso cardiaco. Al trattamento di base 
sono stati aggiunti in maniera randomizzata valsartan o altri farmaci (esclusi sartani). Lo studio è stato interrotto anticipatamente per evidente superiorità di valsartan $(-39 \%$ degli eventi cardiovascolari e $-40 \%$ di ictus). Nello studio KYOTO [11] sono stati arruolati 3.031 pazienti giapponesi con fattori di rischio e con ipertensione non controllata da uno o due farmaci. Alla terapia di base sono stati aggiunti altri antipertensivi (escludendo sartani e aceinibitori) oppure valsartan. Anche questo studio è stato interrotto anticipatamente per manifesta superiorità di valsartan nella riduzione degli endpoint. Valsartan è risultato migliore anche rispetto all'incidenza di nuovi casi di diabete. Questi benefici sono stati osservati nonostante gli effetti sulla pressione arteriosa siano stati simili nei due gruppi, il che conferma che i benefici di valsartan non sono interamente dipendenti dalla sua azione antipertensiva.

Un'analisi cumulativa degli studi JIKEI e KYOTO [12] mostra come valsartan riduca gli eventi cerebrovascolari del $42 \%$, l'angina del $38 \%$, lo scompenso cardiaco del $43 \%$ e l'insufficienza renale del $41 \%$. È interessante rilevare che l'impatto di valsartan sull'ictus $(-42 \%)$ è superiore a quello osservato con losartan nello studio LIFE (-25\%), anche se occorre sottolineare che nella popolazione giapponese (arruolata negli studi JIKEI e KYOTO) l'ictus è l'evento cardiovascolare più frequente, mentre in Europa e Nord America sono più comuni gli eventi coronarici. Particolarmente importante è anche il dato relativo alla prevenzione degli endpoint renali (raddoppio della creatinina e ingresso in dialisi) che risulta più efficace di quella rilevata con losartan nello studio RENAAL.

\section{Scompenso cardiaco}

Losartan è stato valutato nello studio ELITE II [13] effettuato in pazienti ultrasessantenni con scompenso cardiaco in classe NYHA II-IV e con frazione d'eiezione $<40 \%$. Lo studio ha confrontato losartan $(50 \mathrm{mg})$ con captopil (50 mg $\mathrm{x} 3$ ) e ha mostrato che losartan non è superiore a captopril rispetto all'endpoint primario o secondario, ma è meglio tollerato. Valsartan è stato valutato nello studio Val-HeFT [14] che ha incluso pazienti di età $>18$ anni con scompenso cardiaco in classe NYHA II-IV e frazione d'eiezione < $40 \%$. Lo studio ha dimostrato che l'aggiunta di valsartan alla terapia standard riduce l'endpoint combinato mortalità + morbidità del 13,2\% e che valsartan è efficace anche verso gli endpoint secondari. Sulla base dei risultati di questi studi attualmente valsartan ha ottenuto l'indicazione nello scompenso cardiaco come alternativa agli ACE inibitori o come terapia in aggiunta agli stessi nei soggetti che non possono utilizzare $\mathrm{i}$ beta-bloccanti, mentre losartan ha indicazione esclusivamente qualora gli ACE inibitori non siano tollerati o siano controindicati.

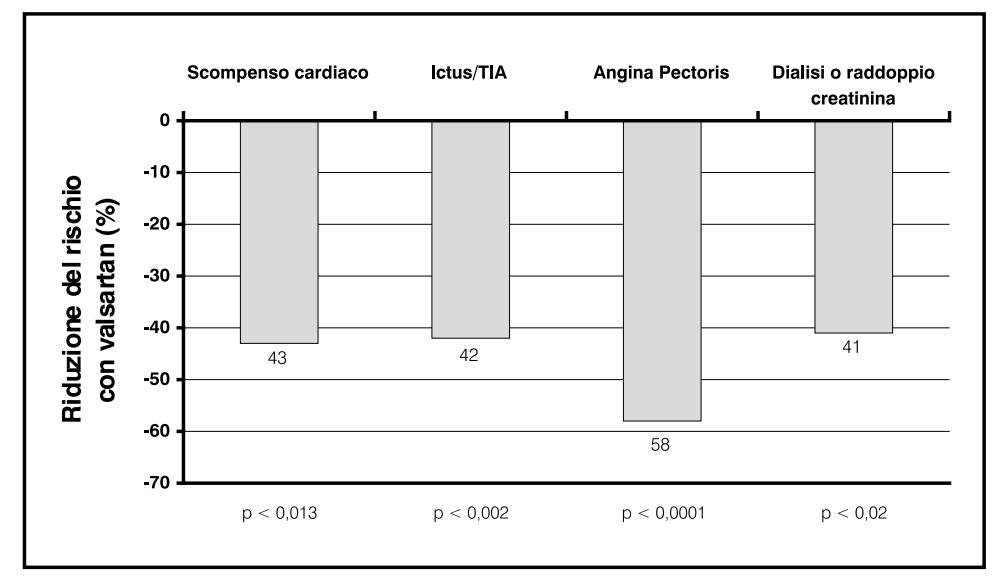

Figura 3

Riduzione degli eventi cardiovascolari in un'analisi comulativa degli studi JIKEI e KYOTO (modificato da Verdecchia, 2010 [12])

$\mathrm{TIA}=$ attacco ischemico transitorio

\section{Infarto miocardico}

Losartan è stato confrontato a captopril nello studio OPTIMAAL [15] in pazienti con infarto miocardico acuto e segni o sintomi di scompenso cardiaco, reinfarto o comparsa di nuova onda Q. Lo studio ha dimostrato che losartan non è superiore a captopril rispetto all'endpoint primario secondario e terziario. Valsartan è stato valutato nello studio VALIANT [16] che ha arruolato 14.500 pazienti con infarto miocardico acuto complicato da scompenso cardiaco, insufficienza ventricolare sinistra (FE $<35 \%)$ o entrambi. L'ipotesi era che valsartan non è inferiore a captopril e l'endpoint primario era la mortalità totale. Lo studio ha confermato l'ipotesi sperimentale dimostrando che valsartan è efficace quanto captopril nel ridurre l'endpoint primario mentre la combinazione dei due aumenta le reazioni avverse senza migliorare la prognosi. Da questi studi è derivato che valsartan, unico tra i sartani, ha indicazione nei pazienti con scompenso cardiaco o disfunzione ventricolare sinistra asintomatica secondaria a infarto miocardico recente, mentre losartan non ha indicazioni nel post-infarto.

\section{Nefropatia}

Lo studio RENAAL [17] ha dimostrato che nei pazienti ipertesi con nefropatia diabetica, a parità di riduzione pressoria, losartan è superiore alla terapia tradizionale nel ridurre $\mathrm{i}$ casi di raddoppio della cretininemia e di insufficienza renale terminale e previene maggiormente i ricoveri per scompenso cardiaco. Valsartan è stato valutato nei pazienti nefropatici in diversi studi: MARVAL 1, MARVAL 2, SMART, DROP. Lo studio MARVAL 1 [18] ha dimostrato che nei soggetti diabetici di tipo 2, ipertesi e non, con microalbuminuria, a parità di valori pressori valsartan produce una maggiore riduzione dell'albuminuria rispetto ad amlodipina. Lo studio MARVAL 2, effettuato in pazienti con 


\section{Figura 4}

Riduzione della PA sistolica ottenuta con dosi crescenti di valsartan in monoterapia o combinato con idroclorotiazide (HCTZ) a vari dosaggi (modificato da Weir, 2007 [22])

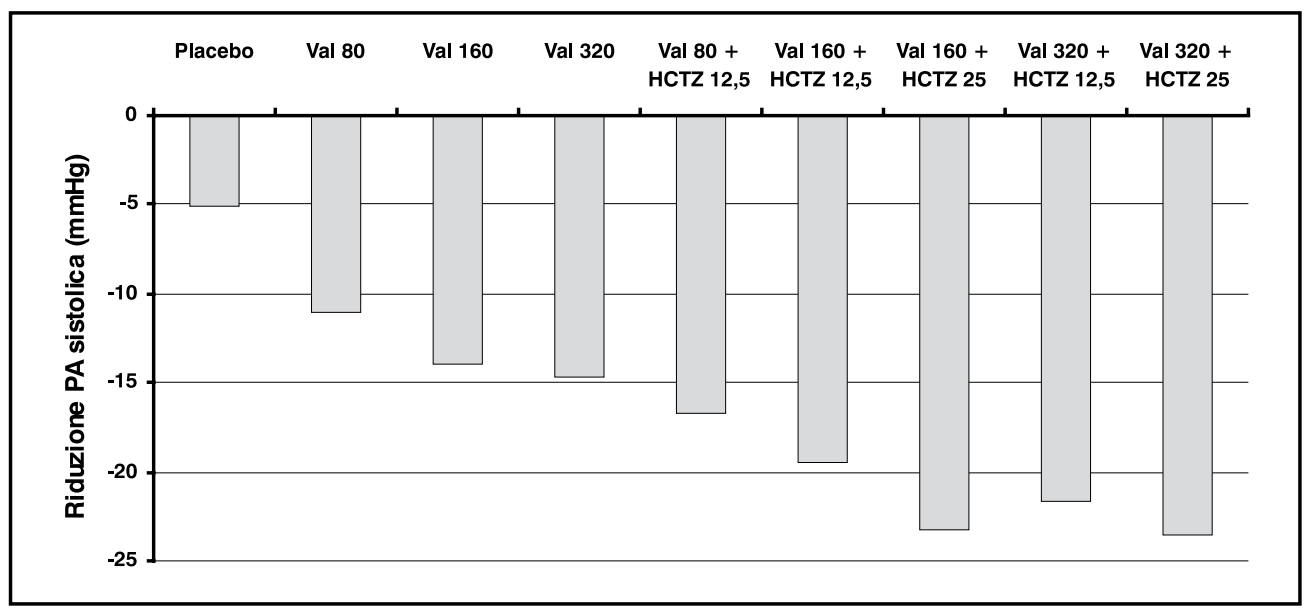

ipertensione sistolica e con microalbuminuria [19] ha dimostrato che l'associazione valsartanidroclorotiazide confrontata con amlodipina, a parità di controllo pressorio, produce una significativa riduzione dell'albuminuria $(-35 \%)$, che viene invece aumentata con amlodipina $(+24 \%)$.

Lo studio SMART [20], ha confrontato valsartan e amlodipina in pazienti ipertesi diabetici e con microalbuminuria. Valsartan è risultato più efficace di amlodipina nel ridurre la microalbuminuria e tale riduzione era significativamente maggiore nel gruppo valsartan con pressione sistolica non controllata, rispetto al gruppo amlodipina con pressione sistolica ben controllata. Ciò dimostra che l'effetto di valsartan è farmaco-dipendente e non pressionedipendente. Lo studio DROP [21] ha valutato l'impatto sull'albuminuria di dosi crescenti di valsartan (fino a $640 \mathrm{mg} / \mathrm{die}$ ) in pazienti con diabete di tipo 2. Lo studio ha evidenziato una riduzione del $50 \%$ della proteinuria, con un calo dose-dipendente e non strettamente correlato alla riduzione pressoria, il che, ancora una volta, suggerisce un effetto non esclusivamente pressione-dipendente. L'insieme di tutti questi studi mostra come valsartan sia un farmaco di provata efficacia in una grande varietà di situazioni cliniche dall'ipertensione, ai soggetti ad alto rischio cardiovascolare, dallo scompenso cardiaco, al post-infarto, e in grado di esercitare un alto grado di protezione anche a livello renale.

\section{La flessibilità di dosaggio}

Valsartan è l'unico sartano disponibile in un vastissimo range di dosaggio sia in monoterapia $(40,80,160,320 \mathrm{mg})$ che in combinazione precostituita con idroclorotiazide (HCTZ) $(12,5$ e $25 \mathrm{mg}$ ). Questa ampia scelta di dosaggio consente di personalizzare la terapia in base alle necessità del singolo paziente. All'aumentare del dosaggio di valsartan infatti aumenta l'entità dell'effetto antipertensivo [22] (Figura 4) e aumenta la percentuale di soggetti che raggiungono il goal terapeutico (Figura 5). Tenendo in considerazione questi risultati, il medico potrà scegliere di volta in volta la dose più adeguata per il suo paziente. In un soggetto con scompenso cardiaco o in un iperteso lieve, in cui si richiede un calo pressorio di pochi $\mathrm{mmHg}$, si potrà iniziare il trattamento con la monoterapia a basso dosaggio, mentre nelle forme più severe di ipertensione si potrà iniziare fin da subito con dosaggi più alti combinati con $\mathrm{HCTZ}$ ottenendo così riduzioni pressorie di oltre $25 \mathrm{mmHg}$ e portando a goal oltre il 70\% dei pazienti [22].

\section{Figura 5}

Percentuale di soggetti a goal con vari dosaggi di valsartan in monoterapia e in combinazione con HCTZ (modificato da Weir, 2007 [22])

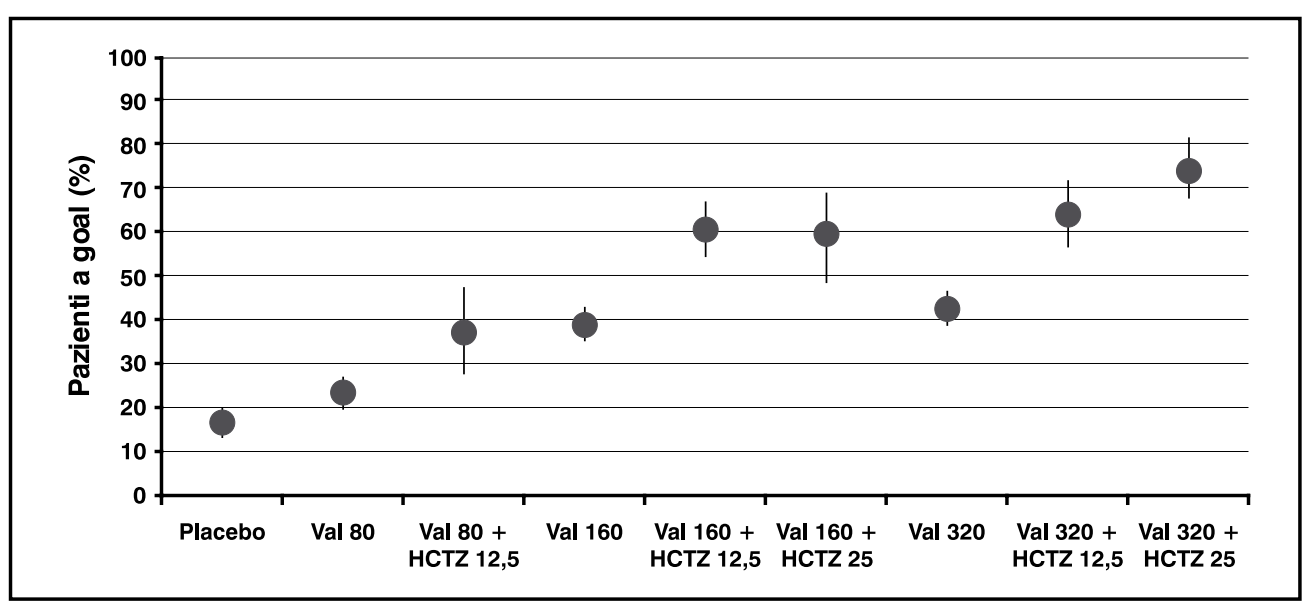


Negli ultimi anni si è andato affermando il concetto di una riduzione pressoria la più rapida possibile. Questo cambiamento d'atteggiamento dal graduale e prudente al veloce $\mathrm{e}$ aggressivo, si basa su una serie di studi [9,2325] che hanno dimostrato come il periodo di tempo intercorrente tra l'inizio della terapia e il raggiungimento degli obiettivi pressori influenza gli esiti cardiovascolari, che sono tanto più favorevoli quanto prima si ottiene il controllo della PA. Il medico invece è stato abituato a un approccio graduale e prudente, con una strategia a gradini, ma il fallimento di tale approccio è sotto gli occhi di tutti date le basse percentuali di soggetti che raggiungono valori pressori soddisfacenti. Un controllo rapido ed efficace dell'ipertensione ha anche un altro risvolto positivo: l'aumento dell'aderenza da parte del paziente. Le due cause fondamentali della scarsa aderenza al trattamento sono infatti le reazioni avverse (che con i sartani sono rarissime) e il mancato controllo pressorio (che demoralizza e demotiva i pazienti).

\section{IL PROBLEMA DI COMPLIANCE E PERSISTENZA: RIFLESSI ECONOMICI}

Uno dei vantaggi principali dei sartani, rispetto alle altre famiglie di farmaci, è costituito dalla tollerabilità, analoga a quella del placebo. Ciò garantisce maggiore aderenza e maggiore persistenza (continuazione nel tempo) al trattamento. Solo l'esecuzione corretta e ininterrotta della terapia garantisce infatti il raggiungimento degli obiettivi della medesima (riduzione degli eventi). I pazienti con livelli di aderenza alla terapia superiori all' $80 \%$ hanno nell'arco di soli 12 mesi, rispetto ai pazienti poco aderenti, una riduzione dell'ictus pari al $22 \%$ [26]. Uno studio effettuato in Italia da medici di famiglia ha confermato questo dato evidenziando che nei pazienti con buona aderenza alla terapia il rischio di eventi cardiovascolari si riduce dal 34 al 50\% a seconda del modello matematico utilizzato [27].

Questo punto è fondamentale anche in relazione al contenimento dei costi sanitari in quanto, nell'ambito delle malattie cardiovascolari oltre il $70 \%$ della spesa è dovuto ai ricoveri ospedalieri (e sale al 94\% nel caso dell'ictus) [28] e quindi, ridurre gli eventi vuol dire ridurre i ricoveri e quindi i costi. Tuttavia, il problema della continuazione regolare della terapia, è tuttora irrisolto ed è probabilmente il fattore che meglio spiega perché meno di un quarto dei pazienti trattati raggiunge valori pressori soddisfacenti. Uno studio eseguito a Pavia (PAPEETE) ha mostrato che, su oltre 65.000 pazienti ipertesi che hanno iniziato per la prima volta un trattamento antipertensivo, solo 1' $11,2 \%$ è ancora in terapia regolare a distanza di un anno [29]. Tuttavia la persistenza è molto diversa a seconda del farmaco che viene prescritto; la letteratura è d'accordo sul fatto che essa è minima coi diuretici e massima coi sartani [30]. Tali dati sono stati confermati anche dallo studio PAPEETE dove il rischio di interrompere il trattamento nei soggetti cui è stato prescritto un diuretico è di oltre nove volte più elevato rispetto a coloro ai quali è stato prescritto un sartano. Anche gli ACE inibitori si associano a un rischio di interruzione più elevato rispetto ai sartani $(\mathrm{RR}=$ $2,10)$ così come i calcio-antagonisti $(R R=2,14)$ e i beta-bloccanti $(R R=1,79)$. L'analisi dei livelli dei persistenza nello studio PAPEETE ha mostrato che nel corso degli anni i soggetti persistenti sono aumentati passando dall' 8 al 13\% e che tale aumento è avvenuto parallelamente a quello delle prescrizioni di sartani, cresciute dal 12 al $15 \%$. Ciò conferma che l'utilizzo su più larga scala dei sartani contribuisce in maniera sostanziale al miglioramento dell'aderenza al trattamento e, quindi, al raggiungimento degli obiettivi della terapia. Lo studio PAPEETE ha inoltre dimostrato che i livelli di persistenza differiscono tra sartano e sartano (Figura 6). I livelli di persistenza più bassi si rilevano infatti con losartan (in monoterapia o in combinazione) mentre i massimi livelli di persistenza si rilevano con valsartan: le probabilità di continuare la terapia con valsartan è del $41,4 \%$ superiore rispetto a losartan.

Come ricordato prima, la non-persistenza genera costi enormi in quanto consuma risorse

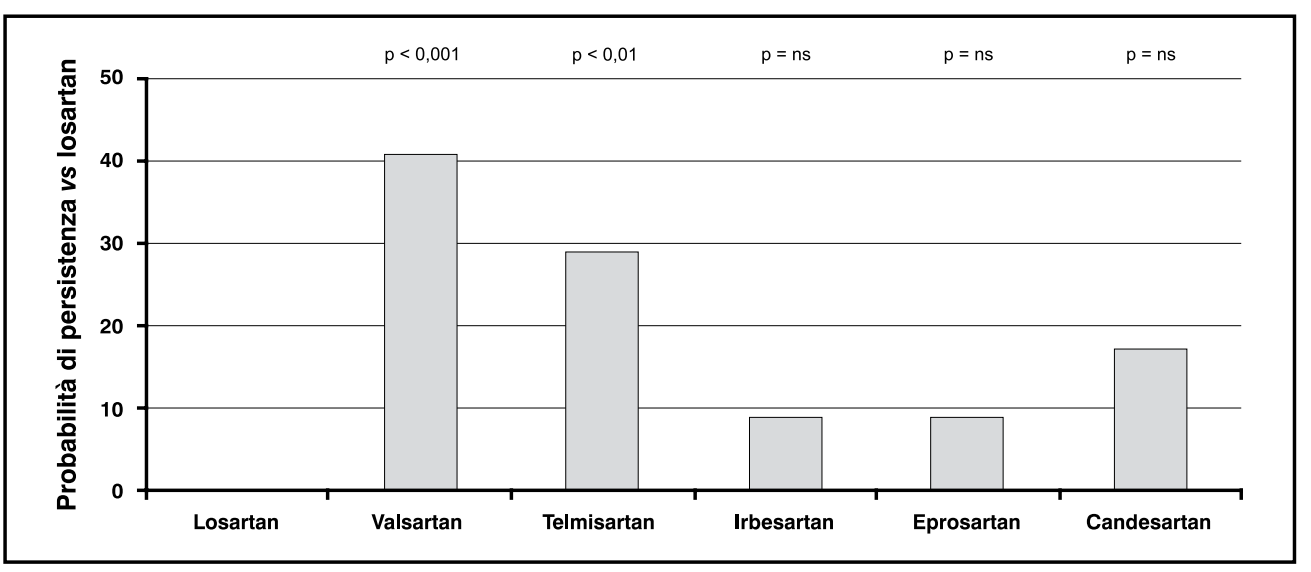

Figura 6

Probabilità di maggiore persistenza dopo un anno di trattamento con vari sartani (in monoterapia o in combinazione precostituita con diuretico) rispetto a losartan che è il sartano con $i$ livelli più bassi di persistenza. Le probabilità sono significativamente maggiori con valsartan $(+41 \%)$ e con telmisartan $(+29 \%)$, ma non con gli altri sartani (modificato da Costa, 2009 [29]) 


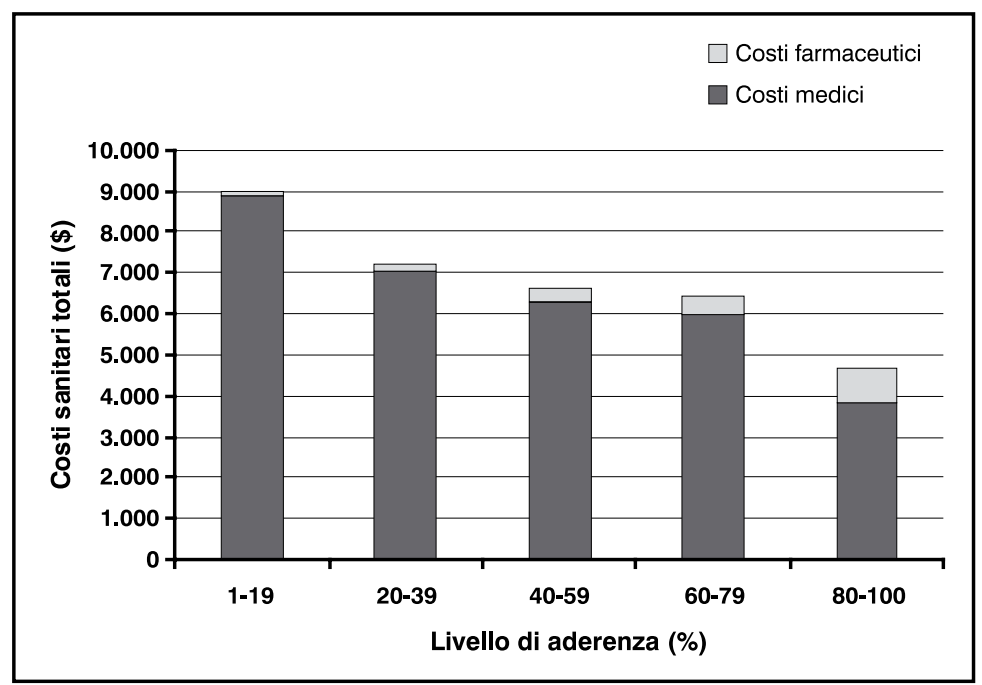

Figura 7

Costi sanitari a seconda del livello di aderenza alla terapia. All'aumentare dell'aderenza cresce la spesa farmaceutica, ma si riducono i costi sanitari. Nei soggetti con elevata aderenza (80-100\%) la spesa per i farmaci è più elevata, ma i costi totali sono la metà di quelli rilevati nei soggetti con aderenza bassissima $(1-19 \%)$

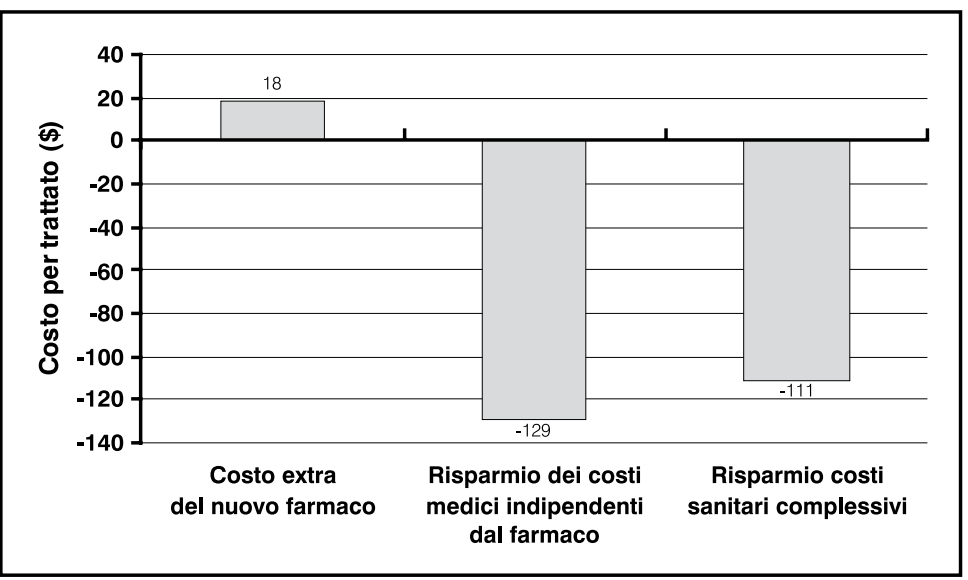

\section{Figura 8}

Analisi dei costi sopportati utilizzando un farmaco nuovo in sostituzione di uno più vecchio e di prezzo più basso. La maggior spesa sopportata per l'acquisto del farmaco viene più che compensata dal risparmio sui costi medici globali (modificato da Lichtenberg, 2002 [32])

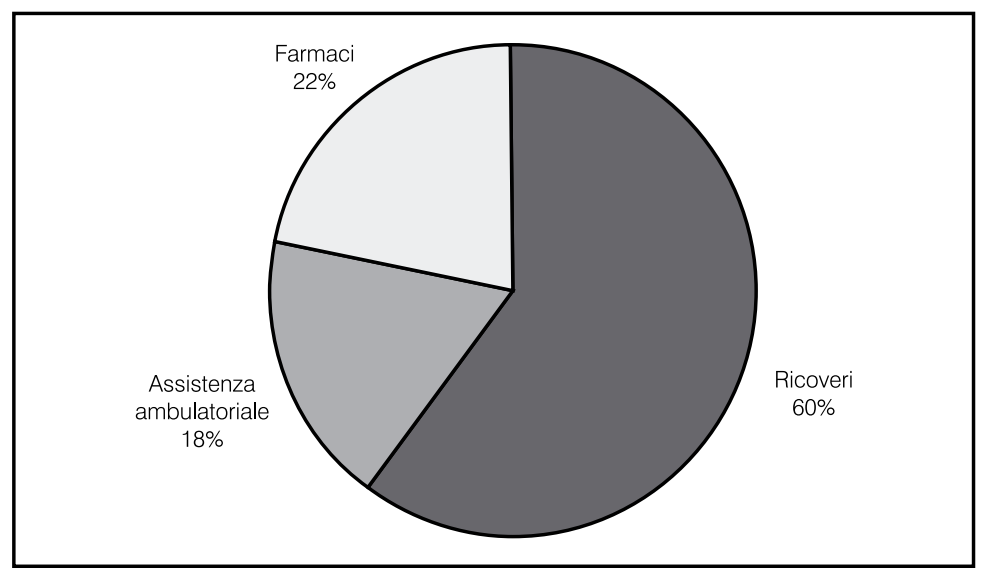

Figura 9

Distribuzione dei costi del diabete tipo II in Italia. I ricoveri in ospedale costituiscono il 60\% del totale della spesa, mentre tra i costi per i farmaci, circa un terzo è costituito dai farmaci cardiovascolari senza produrre i vantaggi attesi dal trattamento (riduzione degli eventi e quindi dei ricoveri). Nella sola ASL di Pavia sono stati spesi sei milioni e mezzo di euro per pazienti che non hanno continuato regolarmente la terapia [29]. È uno spreco immenso di risorse che potrebbe in gran parte essere evitato utilizzando maggiormente i sartani che garantiscono i massimi livelli di persistenza e il cui uso invece viene osteggiato dalle ASL in quanto ritenuti più costosi perchè hanno un prezzo d'acquisto più elevato. Valutare le terapie semplicemente sulla base del prezzo d'acquisto è un approccio metodologicamente sbagliato in quanto il prezzo è solo una delle voci (e non la più rilevante) che compongono i costi totali di un trattamento. Se una terapia garantisce livelli più elevati di aderenza (come nel caso dei sartani), si riducono i ricoveri e i costi medici e anche i costi sanitari totali diventano inferiori. Infatti, come mostrato nella Figura 7, esiste una relazione inversa tra livelli di aderenza al trattamento e costi sanitari totali [31]. Per migliorare l'aderenza nel caso dell'ipertensione bisogna ricorrere ai farmaci più recenti (i sartani) che sono i più tollerabili. Utilizzare farmaci più recenti, come sartani, (e quindi dal prezzo più alto) invece dei più vecchi, non aumenta la spesa [32] ma, al contrario, è stato dimostrato che si ottengono sostanziosi risparmi sulla spesa complessiva (Figura 8). Inoltre, e non dovrebbe mai essere dimenticato, con un incremento di salute per i pazienti. L'insieme di tutte queste osservazioni conferma che migliorare l'aderenza al trattamento significa ridurre gli eventi e quindi i costi. Una politica sanitaria corretta dovrebbe quindi tenere in seria considerazione questi che sono aspetti basilari di economia sanitaria e focalizzarsi sulla necessità di attuare tutte la strategie in grado di migliorare la compliance dei pazienti.

\section{VALSARTAN E PREVENZIONE DEL DIABETE}

Un altro dato rilevante dal punto di visita della salute pubblica e dei costi sanitari, è relativo alla minore incidenza di nuovi casi di diabete osservati con valsartan nel corso di studi come il VALUE ed il KYOTO [9,11]. Il dato, oltre alla ovvia rilevanza clinica, ha importanti risvolti economici. Il diabete infatti è una patologia molto dispendiosa, che in Italia costa oltre 5 miliardi di euro all' anno, che assorbe il 6,65\% di tutta la spesa sanitaria in cui almeno il $60 \%$ della spesa dipende dai ricoveri ospedalieri [33] (Figura 9). I costi, che sono pari a $1.145 € /$ anno nei diabetici non complicati crescono a $5.400 € /$ anno nei pazienti che hanno sviluppato complicanze macro e microvascolari. Ed è a tutti noto come sia molto difficile impedire le complicanze quando il diabete si è già manifestato (Figura 10). Prevenirlo è quindi estremamente importante anche dal punto di vista economico e le capacità di valsartan non devono essere sottovalutate. 


\section{RUOLO DEI FARMACI GENERICI NEL CONTENIMENTO DELLA SPESA FARMACEUTICA}

Di recente, con la scadenza di brevetto di losartan, nell'ottica della riduzione della spesa farmaceutica, i medici sono stati spronati a ricorre a losartan generico. In realtà la riduzione della spesa farmaceutica a carico del SSN viene esclusivamente attuata dalla riduzione automatica del prezzo del farmaco brand, mentre l'utilizzazione del generico non riduce la spesa in quanto la differenza di prezzo tra brand e generico viene pagata direttamente dai pazienti. Losartan poi, dai vari studi su esposti, si è dimostrato in molti casi non all'altezza di valsartan. Ma il vero problema che dobbiamo risolvere è relativo ai dubbi relativi alla reale equivalenza terapeutica dei farmaci generici che sono molti e riconducibili ad alcuni punti fondamentali.

Innanzitutto la bioequivalenza per il generico viene accettata per una variazione compresa tra l' 80 e il $125 \%$ rispetto al brand. Questi margini sono probabilmente troppo ampi per farmaci importanti come i cardiovascolari e comportano il rischio che in alcuni soggetti non si raggiungano livelli plasmatici sufficienti e che in altri si ottengano invece concentrazioni troppo elevate, con rischio di tossicità. Inoltre, i test di bioequivalenza sono quasi sempre effettuati su volontari sani, e non su pazienti, dopo la somministrazione di una sola dose, e utilizzando casistiche molto ridotte. Mancano quindi del tutto dati relativi a pazienti trattati cronicamente o con riduzione della funzione epatica o renale. I farmaci generici inoltre non sono tenuti al rispetto della galenica e spesso utlizzano eccipienti differenti e ciò può impattare negativamente sulla tollerabilità.

Esiste poi il rischio reale che continui cambiamenti del tipo di farmaco generico, (possibili perchè la gestione del generico è ora affidata al farmacista) portino a importanti variazioni dei livelli plasmatici a seconda del generico che gli viene consegnato. Un recente articolo pubblicato su una prestigiosa rivista inglese [34], ha sottolineato i rischi impliciti nel sostituire un farmaco brand con un generico, evidenziando

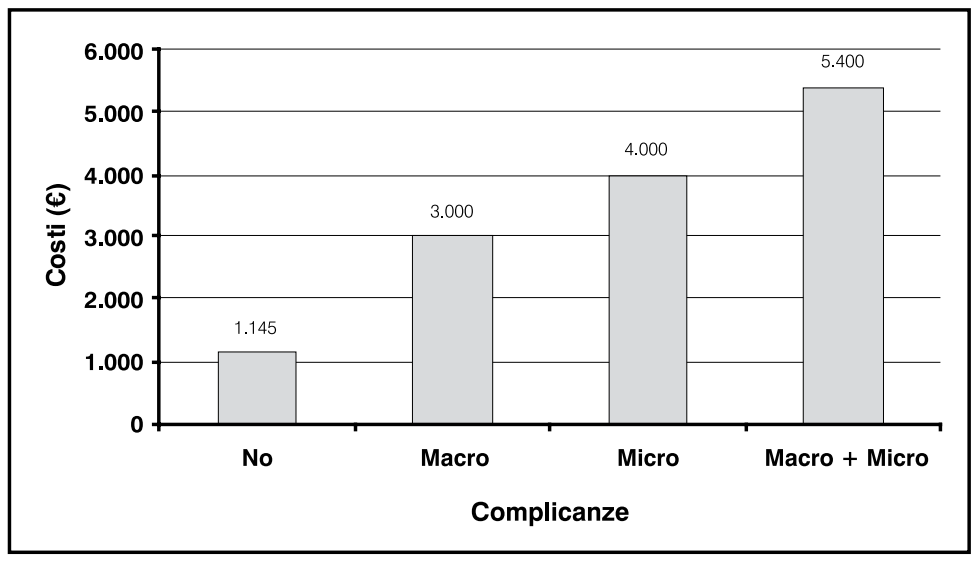

Figura 10

Differenza dei costi del diabete a seconda che siano presenti o meno complicanze macro- e/o microvascolari

anche che tale tipo di operazione comporta sempre dei costi che possono annullare i risparmi legati all'impiego del generico. L'articolo ha anche evidenziato che, nel caso dei sartani, non ci sono garanzie rispetto alla loro reale equivalenza clinica con i farmaci brand.

\section{CONCLUSIONI}

Valsartan, oltre a esercitare un effetto antipertensivo superiore a quello di losartan, è insieme a quest'ultimo, il sartano con il maggior numero di indicazioni cliniche e ciò grazie ai numerosi studi che ne hanno dimostrato l'efficacia in molte patologie, dall'ipertensione all' infarto, dallo scompenso cardiaco, alla nefropatia. Valsartan è in grado di ridurre l'incidenza di nuovi casi di diabete, e garantisce tra i sartani, che pure da questo punto di vista sono in assoluto $\mathrm{i}$ farmaci antipertensivi migliori, $\mathrm{i}$ livelli più alti di persistenza al trattamento. Dato che è dimostrato che la frequenza di eventi cardiovascolari diminuisce sensibilmente all'aumentare dell'aderenza alla terapia, e che i costi sanitari delle malattie cardiovascolari sono in massima parte dipendenti dalla ospedalizzazione, appare evidente che l'utilizzo dei sartani, e di valsartan in particolare, migliorando l'aderenza al trattamento porta a una riduzione dei costi sanitari globali.

\section{BIBLIOGRAFIA}

1. Cheung BM, Cheung GT, Lauder IJ, Lau CP, Kumana CR. Meta-analysis of large outcome trials of angiotensin receptor blockers in hypertension. J Hum Hypertens 2006; 20: 37-43

2. Lee VC, Rhew DC, Dylan M, Badamgarav E, Braunstein GD, Weingarten SR. Meta-Analysis: AngiotensinReceptor Blockers in Chronic Heart Failure and High-Risk Acute Myocardial Infarction. Ann Intern Med 2004; 141: 693-704

3. Siebenhofer A, Plank J, Horvath K, Berghold A, Sutton AJ, Sommer R et al. Angiotensin receptor blockers as antihypertensive treatment for patients with diabetes mellitus: meta-analysis of controlled double-blind randomized trials. Diabet Med 2004; 21: 18-25 
4. Strippoli GF, Craig M, Deeks JJ, Schena FP, Craig JC. Effects of angiotensin converting enzyme inhibitors and angiotensin II receptor antagonists on mortality and renal outcomes in diabetic nephropathy: systematic review. BMJ 2004; 329: 828-38

5. Nixon RM, Müller E, Lowy A, Falvey H. Valsartan vs. other angiotensin II receptor blockers in the treatment of hypertension: a meta-analytical approach. Int J Clin Pract 2009; 63: 766-75

6. Lawes CM, Bennett DA, Feigin VL, Rodgers A. Blood pressure and stroke an overview of published reviews. Stroke 2004; 35: 776-85

7. Dahlöf B, Devereux RB, Kjeldsen SE, Julius S, Beevers G, de Faire U et al.; LIFE study group. Cardiovascular morbidity and mortality in the Losartan Intervention For Endpoint reduction in hypertension study (LIFE): a randomised trial against atenolol. Lancet 2002; 359: 995-1003

8. Schillaci G. Percorso diagnostico dei pazienti ipertesi. Elettrocardiogramma. Ital Heart J 2000; 1(Suppl 5): 3941

9. Julius S, Kjeldsen SE, Weber M, Brunner HR, Ekman S, Hansson L et al.; VALUE trial group. Outcomes in hypertensive patients at high cardiovascular risk treated with regimens based on Valsartan or amlodipine: the VALUE randomised trial. Lancet 2004; 363: 2022-31

10. Mochizuki S, Dahlöf B, Shimizu M, Ikewaki K, Yoshikawa M, Taniguchi I et al.; Jikei Heart Study group. Valsartan in a Japanese population with hypertension and other cardiovascular disease (Jikei Heart Study): a randomised, open-label, blinded endpoint morbidity-mortality study. Lancet 2007; 369: 1431-9

11. Sawada T, Yamada H, Dahlöf B, Matsubara H; KYOTO HEART Study Group. Effects of valsartan on morbidity and mortality in uncontrolled hypertensive patients with high cardiovascular risks: KYOTO HEART study. Eur Heart J 2009; 30: 2461-9

12. Verdecchia P, Angeli F, Mazzotta G, Ambrosio G, Reboldi G. Angiotensin receptor blockers in hypertension.New insights from Japan Hypertension Research. Hypertens Res 2010; 33: 394-7

13. Pitt B, Poole-Wilson PA, Segal R, Martinez FA, Dickstein K, Camm AJ et al. Effect of losartan compared with captopril on mortality in patients with symptomatic heart failure: randomised trial--the Losartan Heart Failure Survival Study ELITE II. Lancet 2000; 355: 1582-7

14. Cohn JN, Tognoni G; Valsartan Heart Failure Trial Investigators. A randomized trial of the angiotensin receptor blocker Valsartan in chronic heart failure. N Engl J Med 2001; 345: 1667-75

15. Dickstein K, Kjekshus J; OPTIMAAL Steering Committee of the OPTIMAAL Study Group. Effects of losartan and captopril on mortality and morbidity in high-risk patients after acute myocardial infarction: the OPTIMAAL randomized trial. Lancet 2002; 360: 752-60

16. Pfeffer MA, McMurray JJ, Velazquez EJ, Rouleau JL, Køber L, Maggioni AP et al.; Valsartan in Acute Myocardial Infarction Trial Investigation. Valsartan, captopril, or both in myocardial infarction complicated by heart failure, left ventricular dysfunction, or both. N Engl J Med 2003; 349: 1893-1906

17. Brenner BM, Cooper ME, de Zeeuw D, Keane WF, Mitch WE, Parving HH et al.; RENAAL Study Investigators. Effects of losartan on renal and cardiovascular outcomes in patients with type 2 diabetes and nephropathy. $N$ Engl J Med 2001; 345: 861-9

18. Viberti G, Wheeldon NM; MicroAlbuminuria Reduction With VALsartan (MARVAL) Study Investigators. Microalbuminuria reduction with valsartan in patients with type 2 diabetes mellitus a blood pressure-independent effect. Circulation 2002;106: 672-8

19. Karalliedde J, Smith A, DeAngelis L, Mirenda V, Kandra A, Botha J et al. Valsartan improves arterial stiffness in type 2 diabetes independently of blood pressure lowering. Hypertension 2008; 51: 1617-23

20. Uzu T, Sawaguchi M, Maegawa H, Kashiwagi A; Shiga Microalbuminuria Reduction Trial (SMART) Group. Reduction of microalbuminuria in patients with type 2 diabetes: the Shiga Microalbuminuria Reduction Trial (SMART). Diabetes Care 2007; 30: 1581-3

21. Hollenberg NK, Parving HH, Viberti G, Remuzzi G, Ritter S, Zelenkofske S et al. Albuminuria response to very high-dose valsartan in type 2 diabetes mellitus. J Hypertens 2007; 25: 1921-6

22. Weir MR, Crikelair N, Levy D, Rocha R, Kuturu V, Glazer R. Evaluation of the dose response with valsartan and valsartan/hydrochlorothiazide in patients with essential hypertension. J Clin Hypertens 2007; 9: 103-12

23. Poulter NR, Wedel H, Dahlöf B, Sever PS, Beevers DG, Caulfield M et al.; ASCOT Investigators. Role of blood pressure and other variables in the differential cardiovascular event rates noted in the Anglo-Scandinavian Cardiac Outcomes Trial-Blood Pressure Lowering Arm (ASCOT-BPLA). Lancet 2005; 366: 907-13 
24. Lithell H, Hansson L, Skoog I, Elmfeldt D, Hofman A, Olofsson B et al.; SCOPE Study Group. The Study on Cognition and Prognosis in the Elderly (SCOPE): principal results of a randomized double-blind intervention trial. J Hypertens 2003; 21: 875-86

25. Basile JN, Chrysant S. The importance of early antihypertensive efficacy: the role of angiotensin II receptor blocker therapy. J Hum Hypertens 2006; 20: 169-75

26. Kettani FZ, Dragomir A, Côté R, Roy L, Bérard A, Blais L et al. Impact of a better adherence to antihypertensive agents on cerebrovascular disease for primary prevention. Stroke 2009; 40: 213-20

27. Mazzaglia G, Ambrosioni E, Alacqua M, Filippi A, Sessa E, Immordino V et al. Adherence to antihypertensive medications and cardiovascular morbidity among newly diagnosed hypertensive patients. Circulation 2009; 120: 1598-605

28. Allender S, Scarborough P, Peto, Rayner M. European cardiovascular disease statistics. Brussels: European Heart Network, 2008

29. Costa FV, Degli Esposti L, Cerra C, Veronesi C, Buda S. Trends in prescription and determinants of persistence to antihypertensive therapy. The Papeete Study. High Blood Pressure \& Cardiovascular Prevention 2009; 16 : $167-76$

30. Costa FV, D’Ausilio A, Negrini C, Lopatriello S. Adherence to anti-hypertensive medications:a review and update. High Blood Pressure \& Cardiovascular Prevention 2009: 16: 101-10

31. Sokol MC, McGuigan KA, Verbugge RR, Epstein RS. Impact of medication adherence on hospitalization risk and healthcare cost. Medical Care 2005; 43: 521-30

32. Lichtenberg F. Benefits and Costs of Newer Drugs: An Update. Cambridge: National Bureau of Economic Research, 2002

33. Lucioni C, Garancini MP, Massi-Benedetti M, Mazzi S, Serra G; CODE-2 Italian Advisory Board. The costs of type 2 diabetes mellitus in Italy: a CODE-2 sub-study. Treat Endocrinol 2003; 2: 121-33

34. Johnston A, Stafylas P, Stergiou GS. Effectiveness, safety and cost of drug substitution in hypertension. $\mathrm{Br} J \mathrm{Clin}$ Pharmacol 2010; 70: 320-34 